09

\title{
О площади предельно коротких электромагнитных импульсов в среде для задач конечной области интегрирования
}

\author{
(C) В.Г. Николаев \\ Университет ИТМО, \\ 197101 Санкт-Петербург, Россия \\ e-mail: vladimir.nikolaev@metalab.ifmo.ru \\ Поступила в редакцию 09.07.2021 г. \\ В окончательной редакции 22.09.2021 г. \\ Принята к публикации 19.10.2021 г.
}

Исходя из одномерных уравнений Максвелла электродинамики сплошной среды получены балансные соотношения электрических и магнитных площадей для задач, решаемых в конечной области по пространству и по времени.

Ключевые слова: предельно короткие импульсы, электрическая площадь импульса.

DOI: $10.21883 /$ OS.2022.02.51997.2530-21

При исследовании предельно коротких импульсов света пропадает возможность применять приближение медленно меняющейся огибающей переменной напряженности электрического поля $\mathscr{E}(t)$. С потерей понятия огибающей импульса не работает инструмент площади импульса $\theta=\frac{d_{12}}{\hbar} \int_{-\infty}^{\infty} \mathscr{E}(t) d t$, здесь $d_{12}$ - дипольный момент перехода в двухуровневом атоме, $\hbar-$ постоянная Планка. Но имеет смысл понятие „электрическая площадь“ $S_{E}=\int_{-\infty}^{\infty} E d t$ (в безразмерном виде $s_{e}=\frac{2 d_{12}}{\hbar} S_{E}$ ). В работах [1-4] получено, что величина $S_{E}(z)$ не меняется с изменением пространственной координаты $z$, вдоль которой происходит распространение электромагнитного излучения. В работах $[3,4]$ приведены условия, при которых интегрирование с бесконечными пределами при вычислении $S_{E}(z)$ заменяются конечными пределами $S_{E}=\int_{t_{1}}^{t_{2}} E d t$. Приведем выдержку из работы [3]: „волновой пакет излучения в начальный момент времени расположен в вакууме, в среде поле отсутствует, а через достаточно большой временной промежуток после прохождения пакета поле в любой фиксированной точке пространства исчезает“.

Опираясь на одномерные уравнения Максвелла электродинамики сплошной среды, получим балансные соотношения электрических и магнитных площадей для задач, решаемых в конечной области как по пространству, так и по времени. Для одномерной геометрии задачи распространения электромагнитного излучения в диэлектрике применяем уравнения Максвелла

$$
\begin{gathered}
\frac{\partial E}{\partial z}+\frac{1}{c} \frac{\partial H}{\partial t}=0, \\
\frac{\partial H}{\partial z}+\frac{1}{c} \frac{\partial E}{\partial t}=-\frac{4 \pi \dot{P}}{c} .
\end{gathered}
$$

Поляризованное излучение распространяется вдоль оси $z$, напряжённость электрического поля $E$ направлена по оси $x$, а магнитного поля $H-$ вдоль оси $y, t-$ время. Система уравнений электродинамики (1), (2) имеет решение в виде электромагнитных волн. Они распространяются как в положительном, так и в отрицательном направлениях.

Задача решается в прямоугольной области $D_{p}$ двух независимых переменных $z$ и $t, z_{L} \leq z \leq z_{R}, t_{B} \leq t \leq t_{E}$. Всё последующее будет справедливо для любой прямоугольной подобласти $D$ области $D_{P}$, ограниченной $z_{1}, z_{2}$ такими, что $z_{L} \leq z_{1}<z_{2} \leq z_{R}$, и $t_{1}, t_{2}-$ $t_{B} \leq t_{1}<t_{2} \leq t_{E}$. Области $D$ приведены на рис. 1 и $2, a$.

Введем обозначения

$$
\begin{gathered}
S_{E, z}=\int_{t_{1}}^{t_{2}} E(z, t) d t, \quad S_{H, z}=\int_{z_{1}}^{z_{2}} \frac{H(z, t)}{c} d z, \\
S_{H, z}=\int_{t_{1}}^{t_{2}} H(z, t) d t \quad S_{E, z}=\int_{z_{1}}^{z_{2}} \frac{E(z, t)}{c} d t, \\
S_{P, z}=\int_{z_{1}}^{z_{2}} \frac{4 \pi P(z, t)}{c} d z .
\end{gathered}
$$

В этих формулах нижние индексы имеют следующий смысл: первый индекс - это подынтегральная функция, второй индекс - это одна из независимых переменных, которая при интегрировании остаётся постоянной. Интегрирование ведется по оставшейся независимой переменной.

Тогда для любой подобласти $D$ справедливы следующие два утверждения:

$$
\begin{gathered}
\left(S_{E, z_{2}}-S_{E, z_{1}}\right)+\left(S_{H, t_{2}}-S_{H, t_{1}}\right)=0, \\
\left(S_{H, z_{2}}-S_{H, z_{1}}\right)+\left(S_{E, t_{2}}-S_{E, t_{1}}+S_{P, t_{2}}-S_{P, t_{1}}\right)=0 .
\end{gathered}
$$


Докажем первое из них. Возьмем по области двойной интеграл от левой части (1) и применим формулу Грина. Получим

$$
\iint_{D_{K}}\left(\frac{\partial E}{\partial z}+\frac{1}{c} \frac{\partial H}{\partial t}\right) d z d t=\oint_{K}\left(-\frac{1}{c} H d z+E d t\right) .
$$

Подынтегральная функция, стоящая в левой части этой формулы, равна нулю, следовательно, двойной интеграл равен нулю. Значит правый криволинейный интеграл тоже равен нулю. Распишем криволинейный интеграл в правой части (7) с учетом направления интегрирования:

$$
\begin{gathered}
\oint_{K}\left(-\frac{1}{c} H d z+E d t\right)=\int_{t_{1}}^{t_{2}} E\left(z_{2}, t\right) d t-\int_{t_{1}}^{t_{2}} E\left(z_{1}, t\right) d t \\
+\int_{z_{1}}^{z_{2}} \frac{H\left(z, t_{2}\right)}{c} d z-\int_{z_{1}}^{z_{2}} \frac{H\left(z, t_{1}\right)}{c} d z=0 .
\end{gathered}
$$

С учетом (3) получаем (5).

Для доказательства справедливости (6) преобразуем (2) к следующему виду:

$$
\frac{\partial H}{\partial z}+\frac{1}{c} \frac{\partial(E+4 \pi P)}{\partial t}=0
$$

Дальнейшее доказательство проводим так же, как и доказательство (5). Выражение в скобках в (8) это есть вектор электрической индукции $D=E+4 \pi P$, что позволяет записать (6) в коротком виде

$$
\left(S_{H, z_{2}}-S_{H, z_{1}}\right)+\left(S_{D, t_{2}}-S_{D, t_{1}}\right)=0 .
$$

Здесь $S_{D, t}=\int_{z_{1}}^{z_{2}} \frac{D(z, t)}{c} d z=\int_{z_{1}}^{z_{2}} \frac{E(z, t)+4 \pi P(z, t)}{c} d z$.

Исследователи часто пользуются безразмерными переменными. Для двухуровневой среды введем безразмерные переменные $[5,6] \tau=t / t_{0}, \xi=z / z_{0}, e=$ $=E / E_{0}, h=H / H_{0}, p=P / P_{0}$, здесь $t_{0}=\omega_{0}^{-1}, z_{0}=c / \omega_{0}$, $E_{0}=H_{0}=P_{0}=\hbar \omega_{0} / 2 d$. Уравнения Максвелла приводятся к виду

$$
\frac{\partial e}{\partial \xi}+\frac{\partial h}{\partial \tau}=0, \quad \frac{\partial h}{\partial \xi}+\frac{\partial(e+4 \pi p)}{\partial \tau}=0 .
$$

Проделав вышеописанные действия, получим вместо (5), (6), (9) следующие соотношения:

$$
\begin{gathered}
\left(s_{e, \xi_{2}}-s_{e, \xi_{1}}\right)+\left(s_{h, \tau_{2}}-s_{h, \tau_{1}}\right)=0, \\
\left(s_{h, \xi_{2}}-s_{h, \xi_{1}}\right)+\left(s_{e, \tau_{2}}-s_{e, \tau_{1}}\right)+\left(s_{4 \pi p, \tau_{2}}-s_{4 \pi p \tau_{1}}\right)=0,
\end{gathered}
$$
или

$$
\left(s_{h, \xi_{2}}-s_{h, \xi_{1}}\right)+\left(s_{d, r_{2}}-s_{d, r_{1}}\right)=0 .
$$

Здесь

$$
s_{e, \xi}=\int_{r_{1}}^{r_{2}} e(\xi, \tau) d \tau, \quad s_{h, r}=\int_{\xi_{1}}^{\xi_{2}} h(\xi, \tau) d \xi,
$$

$$
\begin{gathered}
S_{h, \xi}=\int_{\tau_{1}}^{\tau_{2}} h(\xi, \tau) d \tau, \quad s_{e, \tau}=\int_{\xi_{1}}^{\xi_{2}} e(\xi, \tau) d \xi, \\
s_{4 \pi p, \tau}=\int_{\xi_{1}}^{\xi_{2}} 4 \pi p(\xi, \tau) d \xi \\
s_{d, \tau}=\int_{\xi_{1}}^{\xi_{2}}(e(\xi, \tau)+4 \pi p(\xi, \tau)) d \xi=\int_{\xi_{1}}^{\xi_{2}} d(\xi, \tau) d \xi .
\end{gathered}
$$

Введём следующее обозначение: $r_{f, \xi}=\left(s_{f, \xi_{2}}-s_{f, \xi_{1}}\right)$, это выражение есть разность площадей при большем значении $\left(\xi_{2}\right)$ и при меньшем значении $\left(\xi_{1}\right)$ области $D$. Тогда $(10),(11)$ можно записать в коротком виде

$$
\begin{gathered}
r_{e, \xi}+r_{h \tau}=0, \\
r_{h, \xi}+r_{d, \tau}=0 \quad \text { или } \quad r_{h, \xi}+\left(r_{e, \tau}+r_{4 \pi p, \tau}\right)=0 .
\end{gathered}
$$

Из этих соотношений получаем следующее.

Первое следствие. Для того чтобы одна из разностей в равенствах (12), (13) была равна нулю, необходимо и достаточно, чтобы и вторая разность тоже была равна нулю.

Например, при моделировании прохождения излучения в веществе начальное состояние среды полагается невозмущенным, а $\tau_{2}$ берется достаточно большим, чтобы среда пришла в исходное (невозмущенное) состояние, а всё электромагнитное излучение покинуло среду. Тогда $r_{h, \tau}=0, r_{d, \tau}=0$, следовательно, $r_{e, \xi}=0, r_{h, \xi}=0$. Здесь реализуется закон сохранения электрической и магнитной площадей [1-4].

Второе следствие. Если одна из разностей не равна нулю, то и вторая также не равна нулю. Кроме того, эти разности равны по модулю и противоположны по знаку.

Здесь равенства (12), (13) должно выполняться в любой момент $\tau_{2}=\tau$. Равенство должно выполняться даже тогда, когда в среде присутствует электромагнитное поле или поляризация среды.

При численном моделировании мы получаем приближенное решение. Для контроля правильности полученного численным методом решения применяют расчет баланса величин, подпадающих под законы сохранения, например, полной энергии. Под полной энергией понимается сумма энергии электромагнитного излучения и энергии, аккумулируемой веществом при возбуждении среды. Вышеописанное позволяет ввести дополнительно баланс двух величин: $\Delta s_{e}, \Delta s_{h}$ (дисбалансы площадей электрического и магнитного полей),

$$
\Delta s_{e}=\left(r_{e, \xi}+r_{h, \tau}\right)_{n u}, \quad \Delta s_{h}=\left(r_{h, \xi}+r_{d, \tau}\right)_{n u} .
$$

В относительном виде

$$
\delta s_{e}=\frac{\Delta s_{e}}{\max \left(r_{e, \xi}\right)}, \quad \delta s_{h}=\frac{\Delta s_{h}}{\max \left(r_{h, \xi}\right)} .
$$



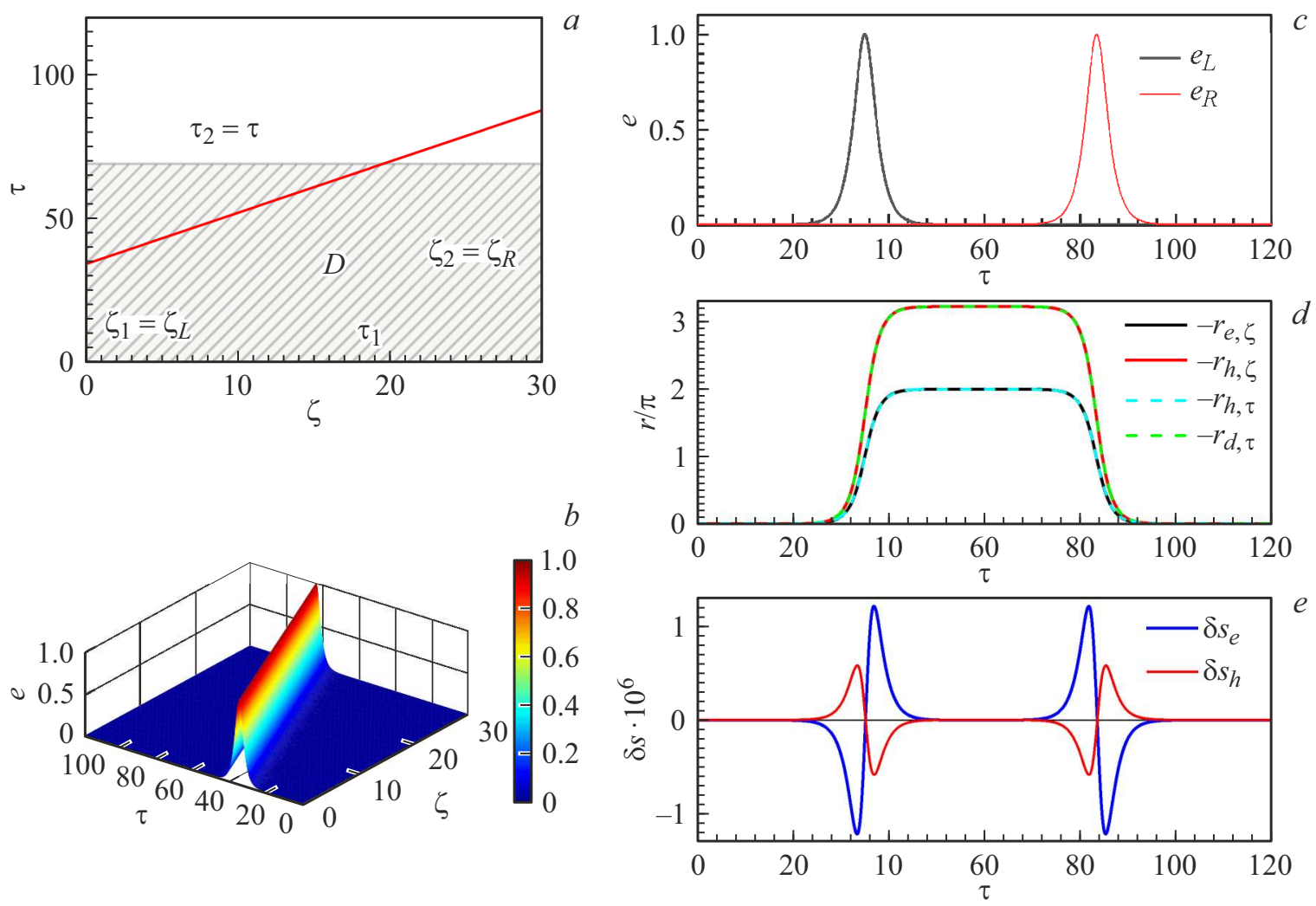

Рис. 1. Задача I. Параметры среды: $N=9 \times 10^{21} \mathrm{~cm}^{-3}, \omega_{0}=2.6909 \times 10^{15}, \lambda=700 \mathrm{~nm}, d=5 D, \alpha=1, \gamma_{1}=\gamma_{2}=0$; длительность импульса $\tau_{p}=2$. (a) Область $D(\tau)$ на плоскости $\xi, \tau$, наклонная прямая - это след максимума импульса. $(b)$ Зависимость $e(\xi, \tau)$. $(c)$ Зависимости $e(\tau)$ на левой и правой границах. (d) Функции $-r_{e \xi}, r_{h r}$, и $-r_{h, \xi}, r_{d, r} .(e)$ Дисбалансы $\delta s_{e}(\tau)$ и $\delta s_{h}(\tau)$.

Нижний индекс у дисбалансов наследуется от первой разности в круглых скобках (14).

Возьмём область $D$, ограниченную по пространственной переменной, $\xi_{1} \leq \xi \leq \xi_{2}$, а по времени $\tau_{1} \leq \tau \leq \tau_{z}$. Здесь $\tau_{z}$ - текущее время задачи, далее будем обозначать его $\tau$. Тогда ,дисбалансы площадей“ будут функцией от переменной $\tau: \delta s_{e}(\tau) \delta s_{h}(\tau)$.

При выводе полученных результатов исходными были только уравнения электродинамики Максвелла, материальные уравнения при выводе не привлекались. Поэтому мы в праве ожидать, что полученные результаты будут справедливы для любой правильной модели среды. В следующих двух примерах в качестве модели среды будем использовать уравнения Блоха для двухуровневого атома: $d s_{1} / d \tau=-s_{2}-\gamma_{2} s_{1}, d s_{2} / d \tau=s_{1}+e s_{3}-\gamma_{2} s_{2}$, $d s_{3} / d \tau=-e s_{2}-\gamma_{1} s_{e 3}$, здесь $\gamma_{i}=\left(\omega_{0} T_{i}\right)^{-1}$. Поляризация $P(t, z)=N d s_{1}$.

Основные результаты решения задачи I и задачи II приведены соответственно на рис. 1 и 2 . Для наглядности параметры задач были подобраны предельно плотными так, что параметр $\alpha=4 \pi N d^{2} /\left(\hbar \omega_{0}\right)$ был по величине порядка $\approx 1-6$.

Первая задача о распространении в плотной среде униполярного импульса самоиндуцированной прозрач- ности. Эта задача имеет аналитическое решение [7]

$$
e=\frac{2}{\tau_{p}} \operatorname{sech}\left(\frac{\tau-\tau_{0}-\xi / v}{\tau_{p}}\right)
$$

В задаче импульс электромагнитного излучения локализован. Можно наблюдать оба сценария, определяемых как первым, так и вторым следствиями.

В качестве второго расчета взята задача из работ $[8,9]$, в которых исследовалось взаимодействие встречных униполярных импульсов самоиндуцированной прозрачности (15) в плотной среде. В результате столкновения двух униполярных импульсов противоположной полярности образуется долгоживущий поляритонный кластер, имеющий двойную пространственную структуру. На графике рис. $2, b$ представлена зависимость $s_{3}(\xi, \tau)$.

Ввиду антисимметрии задачи дисбалансы электрической и магнитной площадей оказались равными нулю тождественно, что говорит о хорошей симметризации численной методики решения. Поэтому область $D$ была взята только по отрицательным значениям $\xi$, график рис. 2, $a$. На графике рис. 2, $c$ приведена только $e_{L}(\tau)$. На правой границе области $D$ из-за антисимметрии задачи $e_{R}(\tau)=e(0, \tau)=0$. На этом графике показана зависимость напряженности магнитного поля при $\xi=0$ в центре симметрии $h_{R}(\tau)$. 

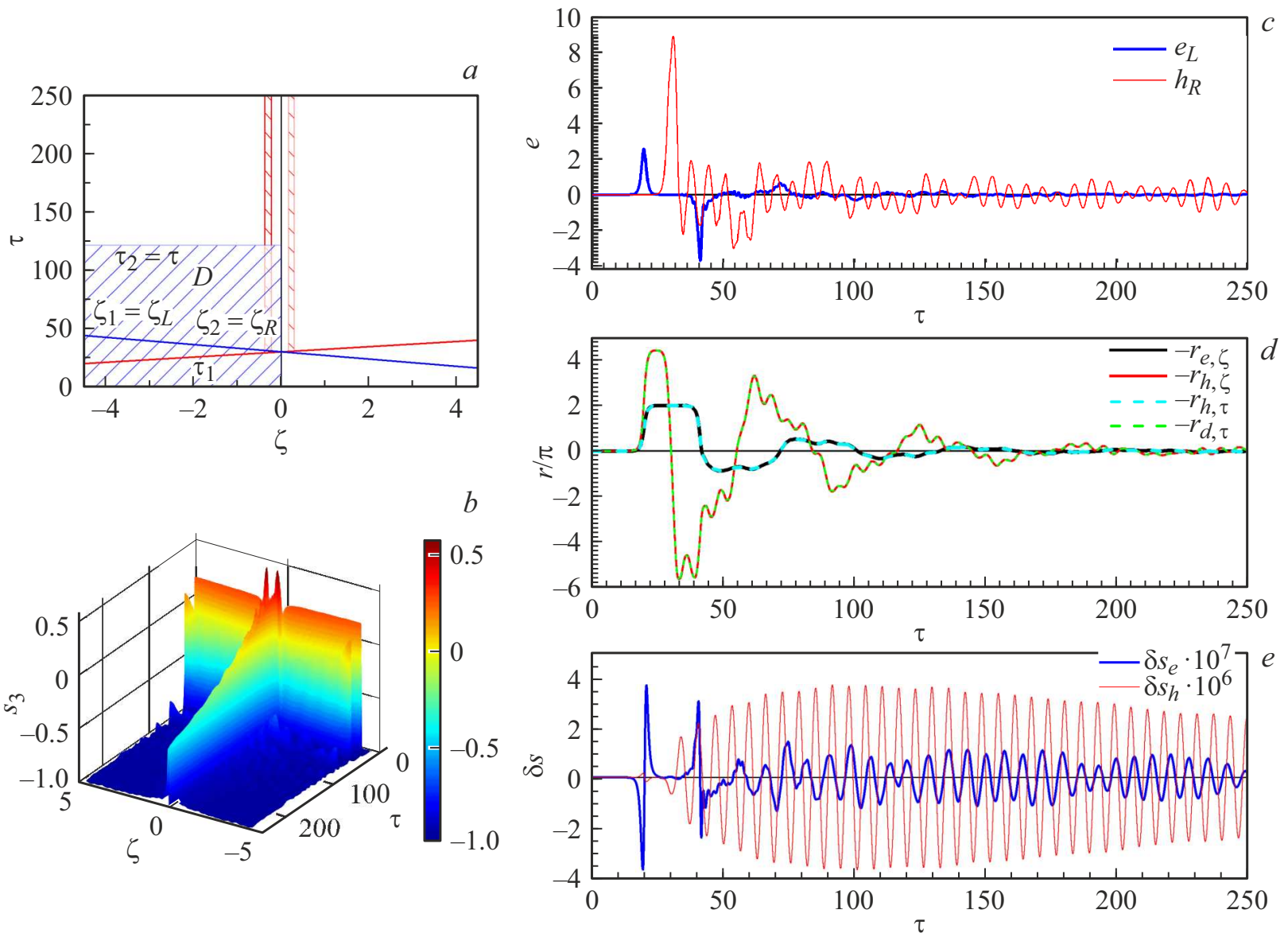

Рис. 2. Задача II. Параметры среды: $N=2.22 \times 10^{21} \mathrm{~cm}^{-3}, \omega_{0}=3.142 \mathrm{fs}^{-1}(\lambda=599.5 \mathrm{~nm}), d=25 D, \alpha=5.260\left(\omega_{c}=2 \mathrm{fs}^{-1}\right)$, $T_{1}=1 \cdot 10^{-13}, T_{2}=0.5 \cdot 10^{-13}$. Длительность импульса $\tau_{p}=0.78$. (a) Область $D(\tau)$ на плоскости $\xi, \tau$, наклонные прямые - это следы максимумов встречных импульсов, узкими вертикальными областями схематично изображены поляритонные кластеры. $(b)$ Зависимость $s_{3}(\xi, \tau)$. (c) Зависимость $e_{L}(\tau)$ на левой границе и зависимость $h_{R}(\tau)$ на правой. $(d)$ Функции $r_{e, \xi}, r_{h, r}$ и $-r_{h, \xi}, r_{d, x} .(e)$ Дисбалансы $\delta s_{e}(\tau)$ и $\delta s_{h}(\tau)$.

Графики $d$ на обоих рисунках демонстрируют как выполняются соотношения (12), (13). Преобразуем (12), (13) к виду $-r_{e, \xi}=r_{h, \tau},-r_{r, \xi}=r_{d, \tau}$, правые и левые части этих соотношений приведены на графиках $d$. Соответствующие пары кривых $-r_{e, \xi}(\tau), r_{h, \tau}(\tau)$ и $-r_{h \xi}(\tau)$, $r_{d, \tau}(\tau)$ на графиках не различимы. Но различия все-таки есть. Это показывают графики $e$, где приведены зависимости относительных дисбалансов площадей $\delta s_{e}(\tau)$ и $\delta s_{h}(\tau)$. Причиной возникновения дисбалансов является использование численных методов решения уравнений Максвелла-Блоха. Вторая задача была рассчитана на трех различных сетках по независимым переменным. Подробность сетки в расчетах менялась на порядок. В таблице приведены значения дисбалансов по энергии $\delta W$ максимумов дисбалансов площадей $\max \delta s_{e}$ и $\max \delta s_{h}$ для задачи II.

Приведенные в таблице данные подтверждают второй порядок точности применяемой методики расчетов. Уменьшение разностной сетки на порядок приводит к уменьшению дисбалансов на два порядка.
Значения дисбалансов по энергии и максимумов дисбалансов площадей электрического и магнитного полей для задачи II

\begin{tabular}{c|c|c|c}
\hline $\begin{array}{c}\text { Шаги разностной } \\
\text { схемы }\end{array}$ & $\delta W$ & $\max \delta s_{e}$ & $\max \delta s_{h}$ \\
\hline $10 \Delta \tau_{2}, 10 \Delta z_{2}$ & $1.26 \cdot 10^{-5}$ & $5 \cdot 10^{-5}$ & $0.37 \cdot 10^{-3}$ \\
$\Delta \tau_{2}, \Delta z_{2}$ & $1.27 \cdot 10^{-7}$ & $4 \cdot 10^{-7}$ & $0.4 \cdot 10^{-5}$ \\
$0.1 \Delta \tau_{2}, 0.1 \Delta z_{2}$ & $1.27 \cdot 10^{-9}$ & $5 \cdot 10^{-9}$ & $0.4 \cdot 10^{-7}$
\end{tabular}

В основном пиковые значения дисбалансов возникают при прохождении импульсов излучения границ среды. Для первой задачи, имеющей аналитическое решение, даже зависимость дисбаланса от времени имеет выражение в аналитическом виде - это производная от гиперболического секанса. Во второй задаче, до момента столкновения встречных импульсов, развитие происходит как в первой задаче. Начиная с момента встречи импульсов, в поведении дисбалансов $\delta s_{e}(\tau)$ и $\delta s_{h}(\tau)$ появляется колебательный характер. Причем амплитуда 
колебаний возрастает и достигает $\max \delta s_{h}=0.4 \cdot 10^{-5}$, но дисбаланс остается вполне приемлемым. Тем более мы всегда можем его уменьшить дроблением разностной сетки.

В заключение подобласть $D$ может состоять из одной, двух или четырех ячеек разностной сетки. В этом случае соотношения (5), (6) могут быть инструментом построения интегро-интерполяционной разностной схемы численного решения задач [10].

\section{Благодарности}

Автор благодарен И.А. Чехонину за обсуждение вопросов, затронутых и работе.

\section{Финансирование работы}

Работа выполнена при поддержке гранта Российского научного фонда № 17-19-01097-П.

\section{Конфликт интересов}

Автор заявляет, что у него нет конфликта интересов.

\section{Список литературы}

[1] Н.Н. Розанов. Опт. и спектр., 107 (5), 761 (2009). [N.N. Rosanov Opt. Spectrosc., 107, 721 (2009)].

[2] Н.Н. Розанов. Диссипативные оптические солитоны от микро- к нано- и атто-. (Физматлит, М. 2011).

[3] Н.Н. Розанов, Р.М. Архипов, М.В. Архипов. УФН, 188 (12), 1347 (2018).

DOI: 10.3367/UFNr.2018.07.038386 [Phys. Usp., 61, 1227 (2018)].

[4] Р.М. Архипов, М.В. Архипов, Н.Н. Розанов. Квант. электрон., 50 (9), 801 (2020). [R.M. Arkhipov, M.V. Arkhipov, N.N. Rosanov. Quant. Electron., 50 (9), 801 (2020)].

[5] J.C. Elbeck, J.D. Gibbon, P.J. Caundrey, R.K. Bullough. J. Phys. A, 6, 1337 (1973).

[6] А.И. Маймистов. Квант. электрон., 30 (4), 287 (2000). [A.I. Maimistov. Quantum Electron., 30, 287 (2000)].

[7] R.K. Bullough, F. Ahmad. Phys. Rev. Lett., 27 (6), 330 (1971).

[8] S.N. Bagaev, V.S. Egorov, V.G. Nikolaev, I.B. Mekhov, I.A. Chekhonin, M.A. Chekhonin. J. Phys.: Conf. Ser., 1124, 051018 (2018). DOI:10.1088/1742-6596/1124/5/051018

[9] S.N. Bagaev, I.B. Mekhov, V.G. Nikolaev, I.A. Chekhonin, M.A. Chekhonin. J. Phys.: Conf. Ser., 1410, 012161 (2019). DOI:10.1088/1742-6596/1410/1/012161

[10] А.А. Самарский. Теория разностных схем. 2 изд. (Наука, M., 1983). 